\title{
The contribution of psychological distress to resting palpitations in patients recovering from severe coronavirus disease 2019
}

\author{
Bing Huang ${ }^{1}$, Hui $\mathrm{Yan}^{2}$, Limei Hu${ }^{1}$, Guipeng Wang ${ }^{2}$, Jing Meng ${ }^{2}$, Wanting $\mathrm{Li}^{3}$, Gang $\mathrm{Liu}^{2}$, \\ Juan Wang ${ }^{2}$, Wenqing Le $^{3}$, and Hong Jiang ${ }^{1}$ \\ ${ }^{1}$ Renmin Hospital of Wuhan University \\ ${ }^{2}$ Fifth Affiliated Hospital of Xinjiang Medical University \\ ${ }^{3}$ Wuhan Hankou Hospital
}

September 16, 2020

\begin{abstract}
Background: Increasing numbers of patients have recovered from severe coronavirus disease 2019 (COVID-19) in Wuhan, China. This study aimed to evaluate the association of psychological distress with resting palpitations in those recovered patients. Methods: In this prospective cohort study, consecutive patients who recovered from severe COVID-19 and complained of resting palpitations were included. Dynamic electrocardiogram (ECG) was continuously monitored for 2 hours while patients were at rest. A survey using palpitation frequency scale and the Hospital Anxiety and Depression Scale (HADS) was administrated to all participants. Results: Of the 289 consecutive patients recovering from severe COVID-19, 24 patients (8.3\%) suffered resting palpitations symptoms, and 22 patients were finally included. Dynamic ECG monitoring showed that 18 (81.8\%) patients had tachyarrhythmias, of which, the most common was sinus tachycardia $(17 / 22,77.3 \%)$. However, patients with sinus tachycardia showed a similar frequency of palpitations episodes compared to those without sinus tachycardia. Anxiety (68.2\%) and depression $(59.1 \%$ ) were prevalent among these recovered patients. Patients with anxiety or depression symptoms were respectively associated with a higher frequency of palpitations episodes than those without. In addition, both HADS-anxiety score $(\mathrm{r}=0.609, \mathrm{P}<0.01)$ and HADS-depression score $(\mathrm{r}=0.516, \mathrm{P}=0.01)$ were positively related to the frequency of palpitations episodes, respectively. Conclusion: Symptom of resting palpitations, manifested mainly by sinus tachycardia, is not uncommon in patients recovering from severe COVID-19. Psychological distress (anxiety and depression) may be responsible, at least in part, for the resting palpitations symptoms.
\end{abstract}

\section{Introduction}

In late December 2019, several cases of pneumonia of unknown origin have been reported in Wuhan, Hubei province, China 1,2. Subsequently, a novel coronavirus was identified by the Chinese Center for Disease Control and Prevention ${ }^{3}$, and was named severe acute respiratory syndrome coronavirus 2 (SARS-CoV-2) by the World Health Organization (WHO). SARS-CoV-2 has robust capacity for human-to-human transmission. A large number of patients were confirmed to been infected by the coronavirus disease 2019 (COVID-19) in Wuhan in the early stage of COVID-19 outbreak ${ }^{4}$. The Chinese government took unprecedented measures such as lockdown of the city, contact tracing, and building Fangcang shelter hospitals to curb the COVID-19 outbreak. Due to the government's great efforts, the COVID-19 epidemic situation in Wuhan was rapidly and effectively controlled. More and more patients recovered from COVID-19 and were discharged from hospitals. However, approximately half of the recovered COVID-19 patients still suffered from various clinical symptoms ${ }^{5}$, of which, symptom of resting palpitations was not rare.

Recently, several studies have focused on the mental health in COVID-19 patients. The results found that a majority of the infected patients experienced various mental disturbances during hospitalization and even 
after discharge ${ }^{6}$. Anxiety and depression were common mental health problems in COVID-19 patients, especially in these severe cases associated with worse outcomes and longer hospital stay ${ }^{7}$.

It is well accepted that psychological distress is one of the most common causes for palpitations symptoms and is associated with the symptom severity ${ }^{8,9}$. Therefore, measures of psychological distress can complement medical assessment in the diagnosis of patients with palpitations ${ }^{10}$. However, few studies have paid attention to the psychological distress in recovered COVID-19 patients with resting palpitations. The aim of the present study was to investigate the prevalence of anxiety and depression, and their association with the frequency of palpitations episodes in patients who recovered from severe COVID-19 and complained of resting palpitations.

\section{Methods \\ Participants}

This single center, prospective, cohort study was performed at the Hankou Hospital in Wuhan City, China. Consecutive patients who recovered from severe COVID-19 and complained of resting palpitations symptoms from February 1 to April 1, 2020, were included.

The severe cases of COVID-19 were defined by the presence of any of the following ${ }^{11}$ : shortness of breath and the respiratory rate $>30$ breaths/min; oxygen saturation [?] 93\%; partial pressure of oxygen to fraction of inspire oxygen ratio [?] $300 \mathrm{mmHg}$; lung infiltrates > 50\% within 24 to 48 hours; respiratory failure; septic shock; and/or multiple organ dysfunction or failure. All the recovered COVID-19 patients met the discharge criteria as follows ${ }^{11}$ : afebrile for at least three days, respiratory symptoms significantly improved, improvement in the radiological abnormalities on chest radiograph or $\mathrm{CT}$, and two consecutive negative SARS-CoV-2 tests more than 24 hours apart. Patients who refused to participate in this study were excluded.

The study protocol was approved by the ethics committee of Renmin Hospital of Wuhan University and Wuhan Hankou Hospital. Written informed consents were obtained from all the individuals who participated in this study.

\section{Data collection}

Patient data, including demographics, medical history, clinical manifestations, laboratory examinations, comorbidities, complications, and hospital stay were collected from the electronic medical records by a trained team of physicians. All the data were independently reviewed. Acute respiratory distress syndrome was defined according to the Berlin Definition ${ }^{12}$. Acute myocardial injury was defined as blood level of cardiac biomarker cTnI increased above the $99^{\text {th }}$ percentile upper reference limit ${ }^{13}$. Acute liver injury was defined as liver enzymes and/or bilirubin were more than twice the upper reference limit ${ }^{13}$. Acute kidney injury was identified according to the Kidney Disease: Improving Global Outcomes definition ${ }^{14}$. Coagulopathy was defined as a 3-s extension of prothrombin time or a 5-s extension of activated partial thromboplastin time ${ }^{13}$.

\section{Electrocardiogram (ECG) monitoring}

A 12-lead ECG monitoring was continuously performed for 2 hours using a Holter monitor (CT-86, Baihui, Hangzhou City, China) in all enrolled participants. All patients lied on the bed quietly with no drink or food since 15 minutes before the monitoring until completion of the monitoring. Holter software was used to analyze the heart rate and arrhythmias. Sinus tachycardia was defined as an increase in sinus rhythm to $>100$ beats/min. Atrial tachycardia was defined as 3 or more consecutive atrial premature contraction. All ECG data were manually examined by two unsuspecting observers and arbitrated by electrophysiologists for any suspicious event classification.

\section{The frequency of palpitations episodes scale}

All participants completed a 7 point rating scale of the frequency of their palpitations episodes: $1=$ rarely (about once or twice a day), $2=$ sometimes (3-5 times a day), $3=$ more than 5 times a day but less than 
once an hour, $4=$ about once or twice an hour, $5=3-5$ times an hour, $6=$ more than 5 times an hour but not sustained, and $7=$ sustained. A higher palpitation score indicates a higher frequency of palpitations episodes.

\section{The Hospital Anxiety and Depression Scale (HADS)}

HADS was a widely used scale for determining anxiety (HADS-A) and depression (HADS-D) in the clinical setting. It comprises of 14 items with a score of 0 to 3 for each item. There is a total score of 21 for anxiety (7 items) and depression (7 items), respectively. The cut-off for clinical anxiety or depression was defined as HADS-A or HADS-D score [?] 8, respectively. A higher score indicates greater anxiety or depression levels.

\section{Statistical analysis}

The continuous and categorical variables are represented as the median (interquartile range [IQR]) and as counts (percentage), respectively. The means for continuous variables were compared using independentsample $t$ tests when the data were normally distributed; otherwise, the Mann-Whitney test was used. The associations of maximum heart rate, HADS-A score, and HADS-D score with palpitation score were analyzed by Pearson's correlation. All data is analyzed using SPSS version 20.0 (SPSS, Chicago, Illinois). The differences were considered statistically significant at a $P$ value of $<0.05$ (two-sided).

\section{Results}

\section{Patient characteristics}

A total of 289 consecutive patients recovering from severe COVID-19 were screened initially, from February 1 to April 1, 2020. Figure 1 shows a flowchart for patient recruitment. Of them, 24 patients (8.3\%) suffered different degrees of palpitation symptom. After excluding 2 patients who refused to provide informed consent, 22 patients were included in this prospective, cohort study.

The details of all the characteristics were presented in the Table1. In total, the median age was 52 years (IQR, 44-62 years), 12 (54.5\%) patients were female, and the median length of hospital stay was 24 days (IQR, 19-28 days). Half of these patients had chronic diseases, including 8 (36.3\%) with hypertension, 3 $(13.6 \%)$ with diabetes, $3(13.6 \%)$ with chronic renal disease, $2(9.1 \%)$ with coronary heart disease, $1(4.5 \%)$ with chronic heart failure, 1 (4.5\%) with chronic obstructive pulmonary disease, and 1 (4.5\%) with cancer. Many patients presented with organ function damage during hospitalization, including $4(18.2 \%)$ with acute respiratory distress syndrome, $4(18.2 \%)$ with acute myocardial injury, $3(13.6 \%)$ with acute liver injury, 2 (9.1\%) with acute renal injury, and $2(9.1 \%)$ with coagulopathy. For treatment, 19 (86.4\%), 17 (77.3\%), $15(68.2 \%)$ and $15(68.2 \%)$ patients, respectively, received antiviral drugs, antibiotics, glucocorticoids, and immunoglobulin. $4(18.2 \%)$ patients used non-invasive ventilator mechanical ventilation, and $1(4.5 \%)$ patient received continuous renal replacement therapy. No patients underwent invasive mechanical ventilation or extracorporeal membrane oxygenation.

\section{Analysis of dynamic ECG monitoring}

Two hours dynamic ECG monitoring showed that 18 (81.8\%) patients had cardiac arrhythmias. Figure 2AD showed representative ECG examples of cardiac arrhythmias. The most common arrhythmia was sinus tachycardia which occurred in $17(77.3 \%)$ patients. The median maximum heart rate during monitoring was $108.5 \mathrm{bpm}$ (IQR, 101-114 bpm). However, patients with sinus tachycardia showed a similar palpitation score with those without sinus tachycardia (median [IQR], 3 [2.5-4] vs 2 [1.5-3.5], $P=0.23$ ) (Figure 3A). In addition, there was no significant relationship between maximum heart rate and palpitation score among the 22 patients $(r=0.323 ; 95 \% \mathrm{CI},-0.11-0.66 ; P=0.14)$ (Figure 3B). Other arrhythmias included atrial premature contraction in $2(9.1 \%)$ patients, atrial tachycardia in $1(4.5 \%)$ patients, and ventricular premature contraction in $1(4.5 \%)$ patients. No bradyarrhythmias were detected.

\section{Palpitation score, HADS score, and the correlations between them}

The medians of the participants' palpitation score, HADS-A score, and HADS-D score were 3 (IQR, 2-4), 9.5 (IQR, 6.75-14), and 8 (IQR, 5-10.25), respectively. Among the 22 patients, 15 (68.2\%) had anxiety 
symptoms (HADS-A [?] 8), and 13 (59.1\%) had depression symptoms (HADS-D [?] 8) (Table 1). Patients with anxiety symptoms (HADS-A [?] 8) were associated with higher palpitation score when compared with those without anxiety (median [IQR], $3[3-4]$ vs $2[1-3], P=0.01$ ) (Figure 4A). Similarly, the palpitation score was also significantly higher in patients with depression (HADS-D [?] 8) than those without depression (median [IQR], 3 [3-4.5] vs $2[1-3], P=0.02)$ (Figure 4B). In addition, both HADS-A score $(r=0.609 ; 95 \%$ CI, $0.25-0.82 ; P<0.01)$ and HADS-D score $(r=0.516 ; 95 \%$ CI, $0.12-0.77 ; P=0.01)$ were positively related to palpitation score (Figure 5A-B).

\section{Discussions}

To our knowledge, this is the first study to investigate the correlation between psychological distress and resting palpitations in patients recovering from severe COVID-19. The major findings of the present study are as follows: (1) symptom of resting palpitations is not uncommon (8.3\%) among patients recovering from severe COVID-19; (2) sinus tachycardia is the most common $(77.3 \%)$ arrhythmia in the recovered patients with resting palpitations; (3) anxiety (68.2\%) and depression (59.1\%) are also prevalent among these patients, and strong correlations were observed between the psychological burden and the frequency of palpitations episodes.

In this prospective cohort study, we found that 24 (8.3\%) of 289 patients had resting palpitations symptoms. It should be noted that all the included patients denied having previous similar episodes of palpitations. Palpitations symptoms occurred during hospitalization and remained after recovery from severe COVID-19.

Palpitation is a common symptom, and often poses a clinical challenge due to the wide differential diagnosis 15. Cardiac arrhythmias and psychological distress are the most common causes in patients presenting with palpitations ${ }^{8}$. The results of two-hour resting ECG monitoring in this study showed that the large majority of the enrolled patients (81.8\%) had cardiac arrhythmias. Among them, the most common was sinus tachycardia (77.3\%). However, patients with sinus tachycardia had a similar frequency of palpitations episodes compared to those without sinus tachycardia, suggesting that there might be other factors, in addition to sinus tachycardia, affecting the palpitations episodes. All the recovered patients did not have any active diseases that might cause sinus tachycardia, such as thyroid dysfunction, haematological disorders, and severe heart failure (left ventricular ejection fraction $<30 \%$ ). Nevertheless, it was a pity that pulmonary function was not evaluated in the present study since this examination was not opened to COVID-19 patients in our hospital, even to those just recovered. Indeed, impaired pulmonary function has been reported in recovered COVID-19 and severe acute respiratory syndrome (SARS) patients, and affected patients' exercise capacity 16,17 . However, none of the enrolled participants complained of any shortness of breath or dyspnea when they were at rest. Therefore, we speculated that the effects of impaired pulmonary function on the resting palpitations symptoms were negligible.

As described above, in addition to arrhythmias, psychological distress is another common cause of palpitations. The prevalence of anxiety and depression was detected by using HADS-A and HADS-D in this study, respectively. The results showed that $68.2 \%$ of patients show anxiety symptoms (HADS-A [?] 8), while $59.1 \%$ show depression symptoms (HADS-D [?] 8). Given the relatively high mortality rate associated with this novel disease, it was not surprising that substantial panic and stress were caused in the early stage of COVID-19 outbreak. Actually, several studies in China have investigated the mental problems in not only COVID-19 patients ${ }^{18,19}$, but also those COVID-19 negative ${ }^{20}$, and the healthcare workers ${ }^{21}$. They also reported that people in Wuhan or Hubei province were prone to higher levels of psychological distress than those in other regions of China ${ }^{20}$. Female sex, disease duration, levels of inflammatory markers and self-perceived illness severity were proposed to be the factors that could predict the severity of patients' mental symptoms ${ }^{19}$. In the present cohort study, all patients recovered from severe COVID-19 in Wuhan, China, with high incidence of various complications (54.5\%) and with very long hospital stay (median [IQR] 24 days [19-28 days]), which might lead to both physical deconditioning and psychological distress.

Psychological distress, such as anxiety and depression-related symptomatology, was frequent among patients with a complaint of palpitation. Alijaniha et al. ${ }^{9}$ showed that $85.4 \%$ of the patients with palpitations 
had psychological disorders and this frequency was twice that of the healthy group (43.6\%). Another study investigating the etiologies of palpitations suggested that up to a third of the patients presenting with palpitations were finally identified to be caused by psychological disorders ${ }^{22}$. On the other hand, symptom of resting palpitations was also one of the most common somatic symptoms associated with anxiety and depression ${ }^{23}$. A recent study by Yuan et al. ${ }^{24}$ found that somatic symptoms are related to the levels of anxiety during the early stage of the COVID-19 outbreak. Consistently with previous studies, we have found that patients with anxiety/depression had a higher frequency of palpitations episodes when compared to those without. Moreover, a close positive correlation between the levels of anxiety/depression and the frequency of palpitations episodes was observed in the present study. These findings suggested that anxiety and depression might be responsible, at least in part, for the resting palpitations symptoms in recovered COVID-19 patients. Importantly, a recent retrospective study from Mayo Clinic showed that anxiety and depression are the most common comorbid conditions in patients with inappropriate sinus tachycardia ${ }^{25}$, proposing a possible link between psychological distress and sinus tachycardia.

\section{Limitations}

Our study has several limitations. First, accumulating evidence has shown that a minority of COVID-19 patients were associated with cardiac complications, including acute myocardial injury, acute myocardial infarction, as well as myocarditis ${ }^{26-27}$, which might result in cardiac structural and electrical changes, causing arrhythmias and palpitations symptoms. However, these effects were not investigated in this study, as only 4 of the enrolled patients had mild elevation of high-sensitive troponin I. Second, since only a small number of patients received psychological guidance due to the relatively limited trained psychiatrist, the therapeutic effects of psychological counseling on palpitations symptoms were not evaluated in the present study. Third, the sample size of the study was too small. Studies with larger populations and in multiple centers are warranted.

\section{Conclusions}

Symptom of resting palpitations, manifested mainly by sinus tachycardia, is not uncommon in patients recovering from severe COVID-19. Anxiety and depression may contribute, at least in part, to the resting palpitations symptoms. Since the accurate etiology leads to an effective treatment, it is highly recommended that psychiatric assessments should be performed in the recovered COVID-19 patients with resting palpitations after a negative evaluation by a cardiologist. In addition, previous studies in recovered SARS patients have shown that palpitations symptoms may last for a long time after discharge ${ }^{27}$. Future studies are warranted to evaluate whether psychological guidance could shorten the course of palpitations symptoms in the recovered COVID-19 patients.

Source of Funding:The foundation of Xinjiang Science and Technology Support Project (2020E0274).

\section{Acknowledgments: None}

\section{References}

1.Huang C, Wang Y, Li X, Ren L, Zhao J, Hu Y, Zhang L, Fan G, Xu J, Gu X, Cheng Z, Yu T, Xia J, Wei Y, Wu W, Xie X, Yin W, Li H, Liu M, Xiao Y, Gao H, Guo L, Xie J, Wang G, Jiang R, Gao Z, Jin Q, Wang J, Cao B. Clinical features of patients infected with 2019 novel coronavirus in Wuhan, China. LANCET. 2020;395(10223):497-506.

2.Chen N, Zhou M, Dong X, Qu J, Gong F, Han Y, Qiu Y, Wang J, Liu Y, Wei Y, Xia J, Yu T, Zhang $\mathrm{X}$, Zhang L. Epidemiological and clinical characteristics of 99 cases of 2019 novel coronavirus pneumonia in Wuhan, China: a descriptive study. LANCET. 2020;395(10223):507-513.

3.Lu R, Zhao X, Li J, Niu P, Yang B, Wu H, Wang W, Song H, Huang B, Zhu N, Bi Y, Ma X, Zhan F, Wang L, Hu T, Zhou H, Hu Z, Zhou W, Zhao L, Chen J, Meng Y, Wang J, Lin Y, Yuan J, Xie Z, Ma J, Liu WJ, Wang D, Xu W, Holmes EC, Gao GF, Wu G, Chen W, Shi W, Tan W. Genomic characterisation 
and epidemiology of 2019 novel coronavirus: implications for virus origins and receptor binding. LANCET. 2020;395(10224):565-574.

4. Guan W, Ni Z, Hu Y, Liang W, Zhong N. Clinical Characteristics of Coronavirus Disease 2019 in China. $N$ Engl J Med . 2020;382:1708-20.

5.Wang X, Xu H, Jiang H, Wang L, Lu C, Wei X, Liu J, Xu S. The Clinical Features and Outcomes of Discharged Coronavirus Disease 2019 PatientsA Prospective Cohort Study. QJM. 2020.

6.Vindegaard N, Benros ME. COVID-19 pandemic and mental health consequences: Systematic review of the current evidence. BRAIN BEHAV IMMUN.2020.

7. Weerahandi H, Hochman KA, Simon E, Blaum C, Chodosh J, Duan E, Garry K, Kahan T, Karmen-Tuohy S, Karpel H, Mendoza F, Prete AM, Quintana L, Rutishauser J, Santos ML, Shah K, Sharma S, Simon E, Stirniman A, Horwitz L. Post-discharge health status and symptoms in patients with severe COVID-19. medRxiv. 2020.

8.Gale CP, Camm AJ. Assessment of palpitations. BMJ. 2016;352:h5649.

9.Alijaniha F, Noorbala A, Afsharypuor S, Naseri M, Fallahi F, Mosaddegh M, Faghih ZS, Sadrai S. Relationship Between Palpitation and Mental Health.Iran Red Crescent Med J. 2016;18(3):e22615.

10.Ehlers A, Mayou RA, Sprigings DC, Birkhead J. Psychological and perceptual factors associated with arrhythmias and benign palpitations.PSYCHOSOM MED. 2000;62(5):693-702.

11. National Health Commission of People's Republic of China. Chinese clinical guidelines for COVID-19 diagnosis and treatment (fifth edition). In Chinese. Available at:

http://www.nhc.gov.cn/yzygj/s7653p/202002/3b09b894ac9b4204a79db5b8912d4440/files/7260301a393845fc87fcf6dd52965ec Accessed February 04, 2020.

12.Ranieri VM, Rubenfeld GD, Thompson BT, Ferguson ND, Caldwell E, Fan E, Camporota L, Slutsky AS. Acute respiratory distress syndrome: the Berlin Definition. JAMA. 2012;307(23):2526-2533.

13.Shang Y, Liu T, Wei Y, Li J, Shao L, Liu M, Zhang Y, Zhao Z, Xu H, Peng Z, Zhou F, Wang X. Scoring systems for predicting mortality for severe patients with COVID-19. EClinicalMedicine. 2020;24:100426.

14.Fliser D, Laville M, Covic A, Fouque D, Vanholder R, Juillard L, Van Biesen W. A European Renal Best Practice (ERBP) position statement on the Kidney Disease Improving Global Outcomes (KDIGO) clinical practice guidelines on acute kidney injury: part 1: definitions, conservative management and contrastinduced nephropathy. Nephrol Dial Transplant.2012;27(12):4263-4272.

15. Thavendiranathan P, Bagai A, Khoo C, Dorian P, Choudhry NK. Does this patient with palpitations have a cardiac arrhythmia? JAMA.2009;302(19):2135-2143.

16. Chen R, Gao Y, Chen M, Jian W, Lei C, Zheng J, Li S. Impaired pulmonary function in discharged patients with COVID-19: more work ahead.EUR RESPIR J. 2020;56(1).

17.Hui DS, Joynt GM, Wong KT, Gomersall CD, Li TS, Antonio G, Ko FW, Chan MC, Chan DP, Tong MW, Rainer TH, Ahuja AT, Cockram CS, Sung JJ. Impact of severe acute respiratory syndrome (SARS) on pulmonary function, functional capacity and quality of life in a cohort of survivors. THORAX. 2005;60(5):401409.

18.Bo HX, Li W, Yang Y, Wang Y, Zhang Q, Cheung T, Wu X, Xiang YT. Posttraumatic stress symptoms and attitude toward crisis mental health services among clinically stable patients with COVID-19 in China. PSYCHOL MED.2020:1-2.

19.Hu Y, Chen Y, Zheng Y, You C, Tan J, Hu L, Zhang Z, Ding L. Factors related to mental health of inpatients with COVID-19 in Wuhan, China. BRAIN BEHAV IMMUN. 2020. 
20. Qiu J, Shen B, Zhao M, Wang Z, Xie B, Xu Y. A nationwide survey of psychological distress among Chinese people in the COVID-19 epidemic: implications and policy recommendations. Gen Psychiatr.2020;33(2):e100213.

21.Lai J, Ma S, Wang Y, Cai Z, Hu J, Wei N, Wu J, Du H, Chen T, Li R, Tan H, Kang L, Yao L, Huang M, Wang H, Wang G, Liu Z, Hu S. Factors Associated With Mental Health Outcomes Among Health Care Workers Exposed to Coronavirus Disease 2019. JAMA Netw Open. 2020;3(3):e203976.

22. Weber BE, Kapoor WN. Evaluation and outcomes of patients with palpitations.AM $J$ MED. 1996;100(2):138-148.

23.Haug TT, Mykletun A, Dahl AA. The association between anxiety, depression, and somatic symptoms in a large population: the HUNT-II study.PSYCHOSOM MED. 2004;66(6):845-851.

24. Yuan S, Liao Z, Huang H, Jiang B, Zhang X, Wang Y, Zhao M. Comparison of the Indicators of Psychological Stress in the Population of Hubei Province and Non-Endemic Provinces in China During Two Weeks During the Coronavirus Disease 2019 (COVID-19) Outbreak in February 2020. Med Sci Monit. 2020;26:e923767.

25. Shabtaie SA, Witt CM, Asirvatham SJ. Natural history and clinical outcomes of inappropriate sinus tachycardia. J Cardiovasc Electrophysiol.2020;31(1):137-143.

26. Stefanini GG, Montorfano M, Trabattoni D, Andreini D, Ferrante G, Ancona M, Metra M, Curello S, Maffeo D, Pero G, Cacucci M, Assanelli E, Bellini B, Russo F, Ielasi A, Tespili M, Danzi GB, Vandoni P, Bollati M, Barbieri L, Oreglia J, Lettieri C, Cremonesi A, Carugo S, Reimers B, Condorelli G, Chieffo A. ST-Elevation Myocardial Infarction in Patients with COVID-19: Clinical and Angiographic Outcomes. Circulation . 2020.

27. Knight DS, Kotecha T, Razvi Y, Chacko L, Brown JT, Jeetley PS, Goldring J, Jacobs M, Lamb LE, Negus R, Wolff A, Moon JC, Xue H, Kellman P, Patel N, Fontana M. COVID-19: Myocardial injury in survivors. Circulation . 2020.

28.Lau ST, Yu WC, Mok NS, Tsui PT, Tong WL, Cheng SW. Tachycardia amongst subjects recovering from severe acute respiratory syndrome (SARS).INT J CARDIOL. 2005;100(1):167-169.

\section{Figure legends}

Figure 1 Flowchart of participant selection . COVID-19=coronavirus disease 2019.

Figure 2 Representative ECG examples of cardiac arrhythmias, including sinus tachycardia (ST) (A), atrial premature contraction (APC) (B), atrial tachycardia (AT) (C), and ventricular premature contraction (VPC) (D).

Figure 3 Frequency of palpitations episodes in patients with vs without sinus tachycardia (ST) (A) and correlation analysis of maximum heart rate and frequency of palpitations episodes (B).

Figure 4 Frequency of palpitations episodes in patients with vs without anxiety (A) or depression (B).

Figure 5 Correlation analysis of anxiety $(A)$ or depression (B) levels and frequency of palpitations episodes.

\section{Hosted file}

Table 1-revised.docx available at https://authorea.com/users/359459/articles/481430-thecontribution-of-psychological-distress-to-resting-palpitations-in-patients-recoveringfrom-severe-coronavirus-disease-2019 
Figure 1

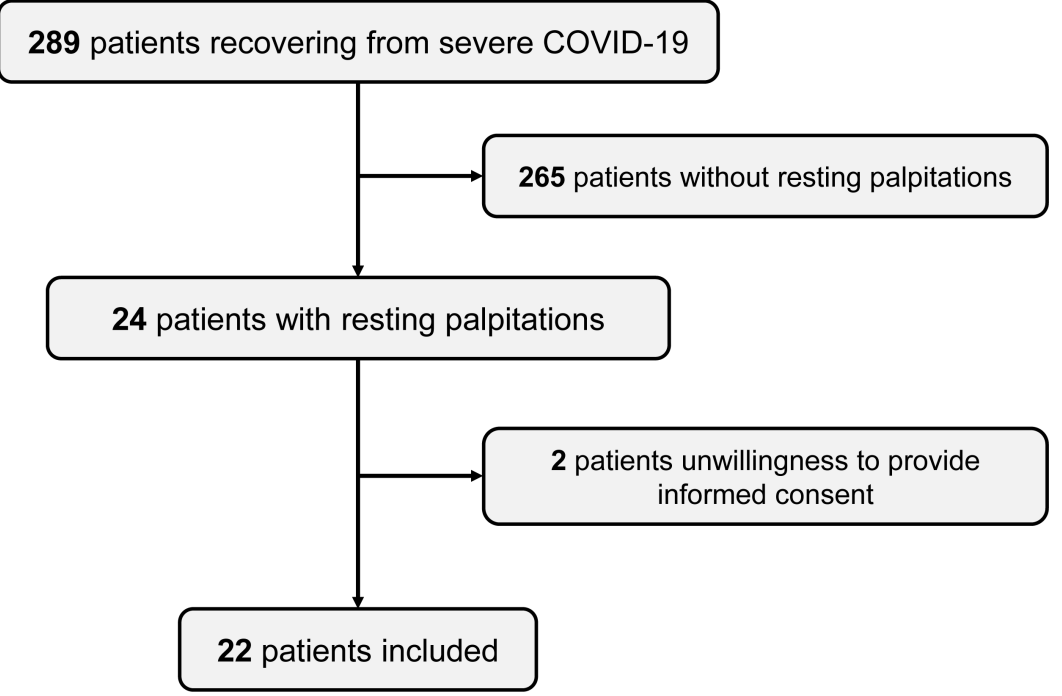

Figure 2

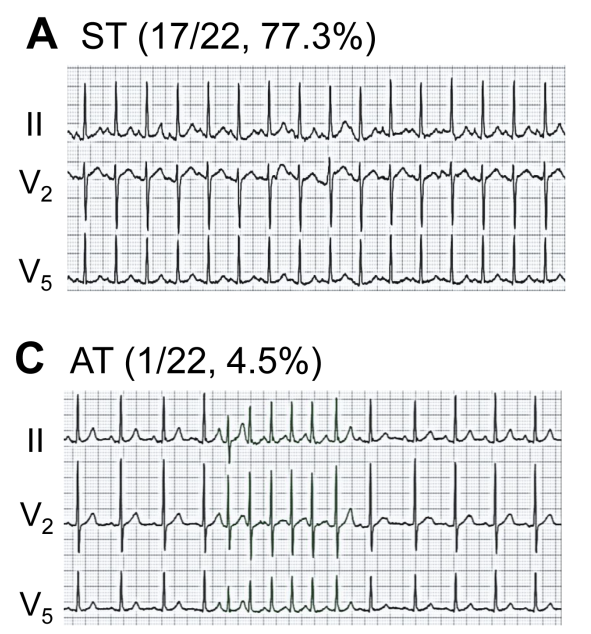

B $\operatorname{APC}(2 / 22,9.1 \%)$

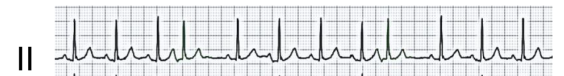

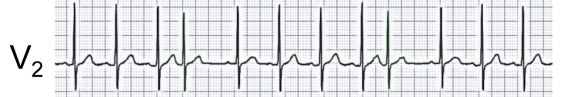

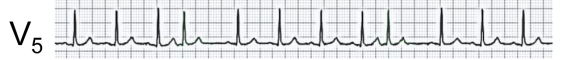

D $\operatorname{VPC}(1 / 22,4.5 \%)$

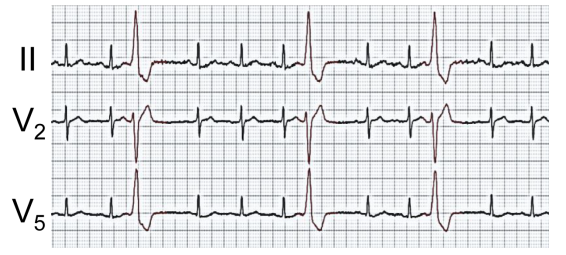


Figure 3
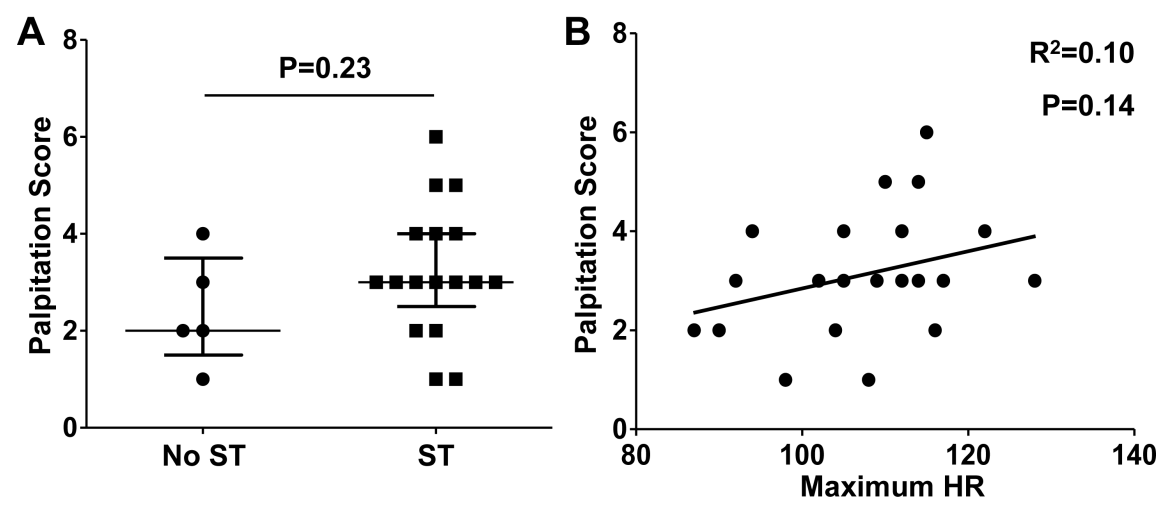

Figure 4
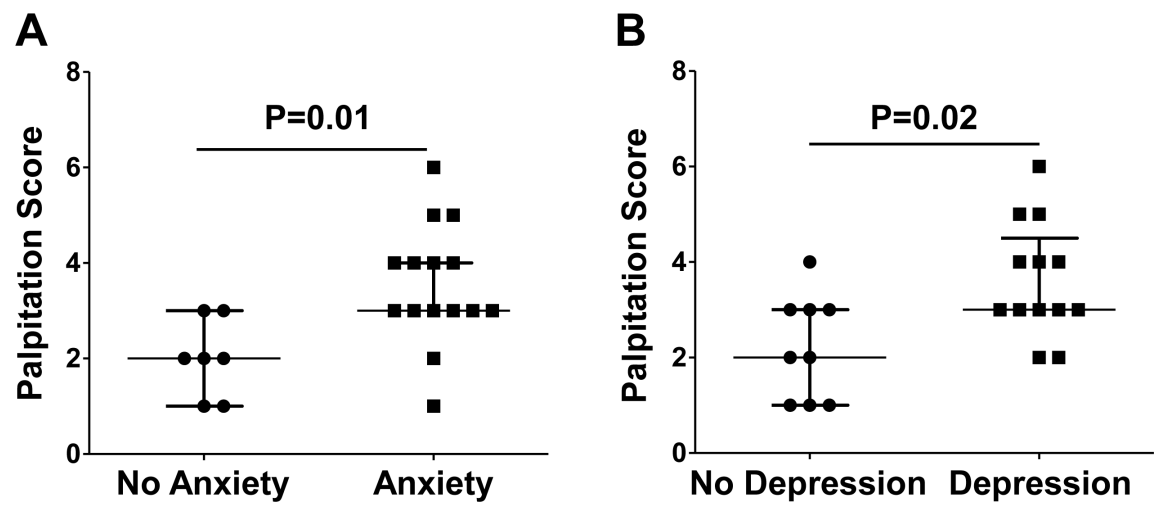

Figure 5
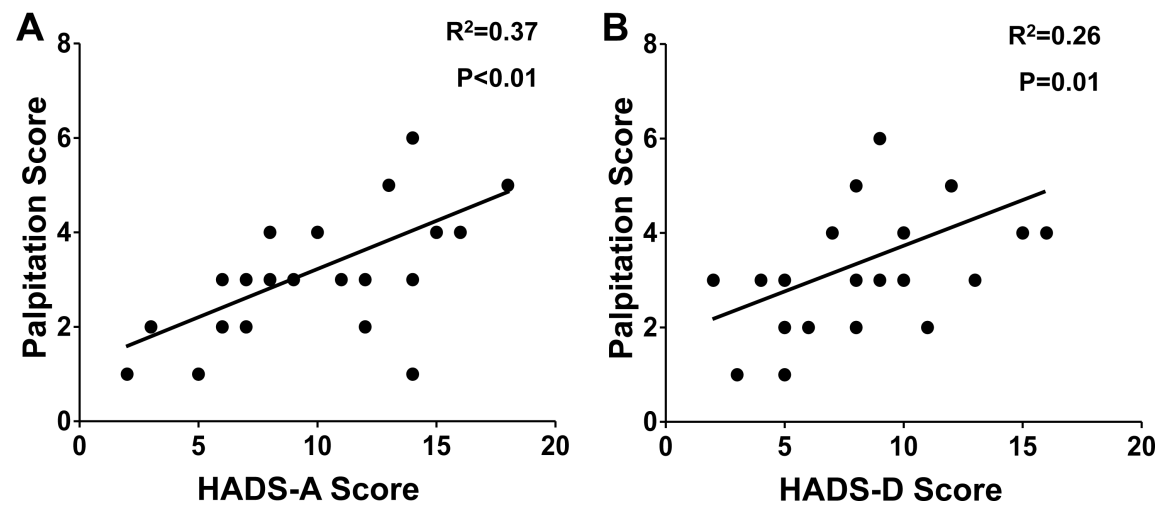